\title{
Simulação com debriefing com vídeo no ensino do exame físico respiratório: Satisfação e a autoconfiança dos estudantes de enfermagem
}

Simulation with debriefing with video in the teaching of the respiratory physical examination: Satisfaction and self-confidence of nursing students

Simulación con debriyfing con video en la enseñanza del examen físico respiratorio: Satisfaccióny autoconfianza de los estudiantes de enfermería

Recebido: 08/06/2021 | Revisado: 16/06/2021 | Aceito: 18/06/2021 | Publicado: 23/06/2021

Patrícia Costa dos Santos da Silva ORCID: https://orcid.org/0000-0001-9643-1865

Universidade Federal de Uberlândia, Brasil E-mail: patriciacosta@ufu.br

Daniela Grotto Alves

ORCID: https://orcid.org/0000-0002-4390-3547 Universidade Federal de Uberlândia, Brasil

E-mail: dani_grotto@hotmail.com

Clesnan Mendes-Rodrigues

ORCID: https://orcid.org/0000-0002-8871-7422

Universidade Federal de Uberlândia, Brasil E-mail: clesnan@hotmail.com

Mariana Ramos

ORCID: https://orcid.org/0000-0003-3230-8424

Universidade Federal de Uberlândia, Brasil E-mail: mari.ramos2009@hotmail.com

Luana Araújo Macedo Scalia

ORCID: https://orcid.org/0000-0003-1000-8738

Universidade Federal de Uberlândia, Brasil

E-mail: luanascalia@ufu.br

Caio César Gonçalves de Holanda

ORCID: https://orcid.org/0000-0003-0339-8635 Centro Universitário IMEPAC Brasil

E-mail: caiocesarholanda@gmail.com

Ricardo Gonçalves de Holanda

ORCID: https://orcid.org/0000-0002-2347-4810

Universidade Federal de Uberlândia, Brasil E-mail: profricardoholanda@ufu.br

Suely Amorim de Araújo

ORCID: https://orcid.org/0000-0001-9234-166X

Universidade Federal de Uberlândia, Brasil E-mail: profasuelyamorim@ufu.br

\begin{abstract}
Resumo
Objetivo: Avaliar a satisfação e a autoconfiança na aprendizagem dos estudantes de Enfermagem que fizeram parte de uma aula experimental sobre ensino do exame físico respiratório, baseada em simulação com debriefing com vídeo. Método: Trata-se de um estudo analítico-descritivo e quantitativo, realizado com uma amostra de 33 estudantes de graduação em Enfermagem da Universidade Federal de Uberlândia que participaram da aula experiencial baseada em simulação e em utilização de um vídeo no debriefing. A coleta de dados foi feita por meio da aplicação da escala de satisfação e da autoconfiança (Student Satisfaction and Self-Confidence in Learning - SSSCL) nos estudantes após a participação na aula. Resultados: Houve predomínio do sexo feminino na amostra com 78,8\% e de estudantes jovens com idade média de 20,91 anos. Em relação à escala de satisfação e de autoconfiança, mostraram-se médias de 4,36 e 4,15 , respectivamente; todos os itens da escala obtiveram média acima de quatro, com exceção do item seis, com média 3,39 , mesmo assim, um valor acima do moderado. Estudantes do sexo feminino tiveram menor satisfação $(B i=-0,451$; $p<0,05)$, quando comparadas aos estudantes masculinos. Já a autoconfiança, não dependeu do perfil dos estudantes. Conclusão: Conclui-se que o uso de simulação com o uso de vídeo no debriefing promove satisfação e autoconfiança na aprendizagem entre os estudantes de Enfermagem.
\end{abstract}

Palavras-chave: Ensino; Sistematização da assistência de enfermagem; Enfermagem; Simulação. 


\begin{abstract}
Objective: To evaluate the satisfaction and self-confidence in the learning of nursing students who were part of an experimental class on teaching the respiratory physical examination, based on simulation with video debriefing. Method: This is an analytical-descriptive and quantitative study, conducted with a sample of 33 undergraduate nursing students from the Federal University of Uberlândia who participated in the experiential class based on simulation and using a video in the debriefing. Data collection was performed by applying the Student Satisfaction and Self-Confidence in Learning (SSSCL) scale to students after participation in the class. Results: There was a predominance of females in the sample with $78.8 \%$ and young students with a mean age of 20.91 years. Regarding the satisfaction and selfconfidence scale, averages of 4.36 and 4.15 , respectively; all items of the scale obtained an average above four, except for item six, with an average of 3.39 , even so, a value above the moderate. Female students had lower satisfaction $(B i$ $=-0.451 ; p<0.05)$, when compared to male students. Self-confidence, on the other hand, did not depend on the profile of the students. Conclusion: It is concluded that the use of simulation with the use of video in the debriefing promotes satisfaction and self-confidence in learning among nursing students.
\end{abstract}

Keywords: Teaching; Systematization of nursing care; Nursing; Simulation technique.

\title{
Resumen
}

Objetivo: Evaluar la satisfacción y la confianza en sí mismos en el aprendizaje de los estudiantes de enfermería que formaban parte de una clase experimental sobre la enseñanza del examen físico respiratorio, con base en la simulación con video debriefing. Método: Se trata de un estudio analítico-descriptivo y cuantitativo, realizado con una muestra de 33 estudiantes de graduación en enfermería de la Universidad Federal de Uberlândia que participaron en la clase experiencial basada en la simulación y utilizando un video en el debriefing. La recolección de datos se realizó aplicando la escala de satisfacción y confianza en sí mismo en el aprendizaje (SSSCL) de los estudiantes después de la participación en la clase. Resultados: Predominó el sexo femenino en la muestra con 78,8\% y los jóvenes estudiantes con edad media de 20,91 años. En cuanto a la escala de satisfacción y autoconfianza, se promediaron promedios de 4,36 y 4,15, respectivamente; todos los ítems de la escala obtuvieron un promedio superior a cuatro, a excepción del ítem seis, con un promedio de 3,39, aun así, un valor por encima del moderado. Las alumnas tuvieron menor satisfacción (Bi $=-0,451 ; p<0,05)$, en comparación con los estudiantes varones. La confianza en sí mismo, por otro lado, no dependía del perfil de los estudiantes. Conclusión: Se concluye que el uso de la simulación con el uso de video en el debriefing promueve la satisfacción y la confianza en sí mismo en el aprendizaje entre los estudiantes de enfermería.

Palabras clave: Enseñanza; Sistematización de la atención de enfermería; Enfermería; Simulación.

\section{Introdução}

Com o passar dos anos torna-se cada vez mais presente o avanço tecnológico e científico na área da saúde (Oliveira, et al., 2014). Desta forma, a atualização constante do conhecimento assim como das metodologias de ensino são imprescindíveis e devem evoluir conforme as informações são processadas. Assim, temos nas metodologias ativas de ensino e aprendizagem uma proposta atraente e que tem a intenção de estimular a visão crítica e criativa dos alunos, distanciando-os dos métodos tradicionais, vinculados apenas ao repasse de informações (Oliveira, et al., 2014).

A preocupação com a formação profissional dos futuros enfermeiros têm sido objeto de diversos estudos (Ali, et al., 2019; Montejano-Lozoya, et al. 2019; Al Gharibi \& Arulappan, 2020; Padilha, et al., 2020). Nestes estudos, destaca-se o uso da simulação como ferramenta de ensino que, por sua vez, vem alcançando reconhecimento e espaço nas universidades do mundo, particularmente nos cursos de graduação em Enfermagem (Oliveira, et al., 2014; Branna, et al., 2016; Johnsen, et al. 2016; Padilha, et al., 2020).

Em situações simuladas os estudantes podem vivenciar situações que melhoram sua autoconfiança, por imitar a realidade (Al Gharibi \& Arulappan, 2020; Padilha, et al., 2020). Além disso, estimula a reflexão e o raciocínio clínico dos alunos, motivando a aprendizagem e favorecendo o aprimoramento das habilidades psicomotoras (Johnsen, et al. 2016). Na simulação o discente pode assimilar o conteúdo de forma mais prazerosa, colocando em prática habilidades técnicas e habilidades de liderança.

Nesse sentido, investigar maneiras pelas quais os estudantes aprendem de forma mais eficiente, a partir do que lhes entusiasmam e que os tornam confiantes, pode representar uma evidência importante para o processo de formação e qualificação profissional para atuarem frente as mudanças e transformações atuais no cenário da saúde (Costa, et al., 2020).Tendo em vista que os estudos científicos apontam o uso da simulação clínica como diferencial das outras metodologias de ensino devido à 
possibilidade da aprendizagem experiencial; focalizada no aluno em ambiente seguro; respaldada por uma reflexão contínua; guiada por um professor (Marmol, et al., 2012; Oliveira, et al., 2014; Al Gharibi \& Arulappan, 2020).

O objetivo deste estudo foi avaliar a satisfação e autoconfiança de estudantes que participaram de uma aula experimental de simulação realística executada no curso de graduação em Enfermagem da Universidade Federal de Uberlândia.

\section{Metodologia}

\subsection{Local de estudo e população}

A presente investigação trata-se de um estudo analítico, descritivo e quantitativo (Polit \& Beck, 2006). A populações eram constituídas por 38 alunos, que cursavam o terceiro, o quarto e o sexto período do curso de graduação em Enfermagem e que utilizaram o laboratório de Enfermagem nas disciplinas: Sistematização da Assistência de Enfermagem (SAE) e Fundamentos de Enfermagem. A amostra foi por conveniência e foi composta por 33 acadêmicos. Como critérios de inclusão foram adotados estudantes maiores de 18 anos de idade, matriculados no curso de graduação em Enfermagem da Universidade Federal de Uberlândia; estar cursando ou ter cursado a disciplina de SAE e os como critério de exclusão os alunos que não tenham participado de todas as etapas da aula experimental e do preenchimento da escala conforme demonstrado na Figura 1.

Figura 1. Fluxo utilizado na aplicação da escala de satisfação e autoconfiança sobre a aula experimental do exame físico respiratório dentro da Sistematização da Assistência de Enfermagem.

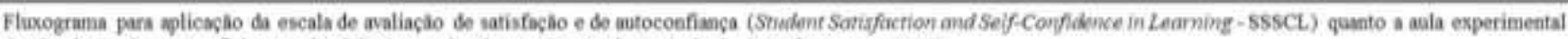
de simulaçào do exame fisico respiratório dentro de Sistematizaç to da Assistêsecis de Enfermagem (SAE).

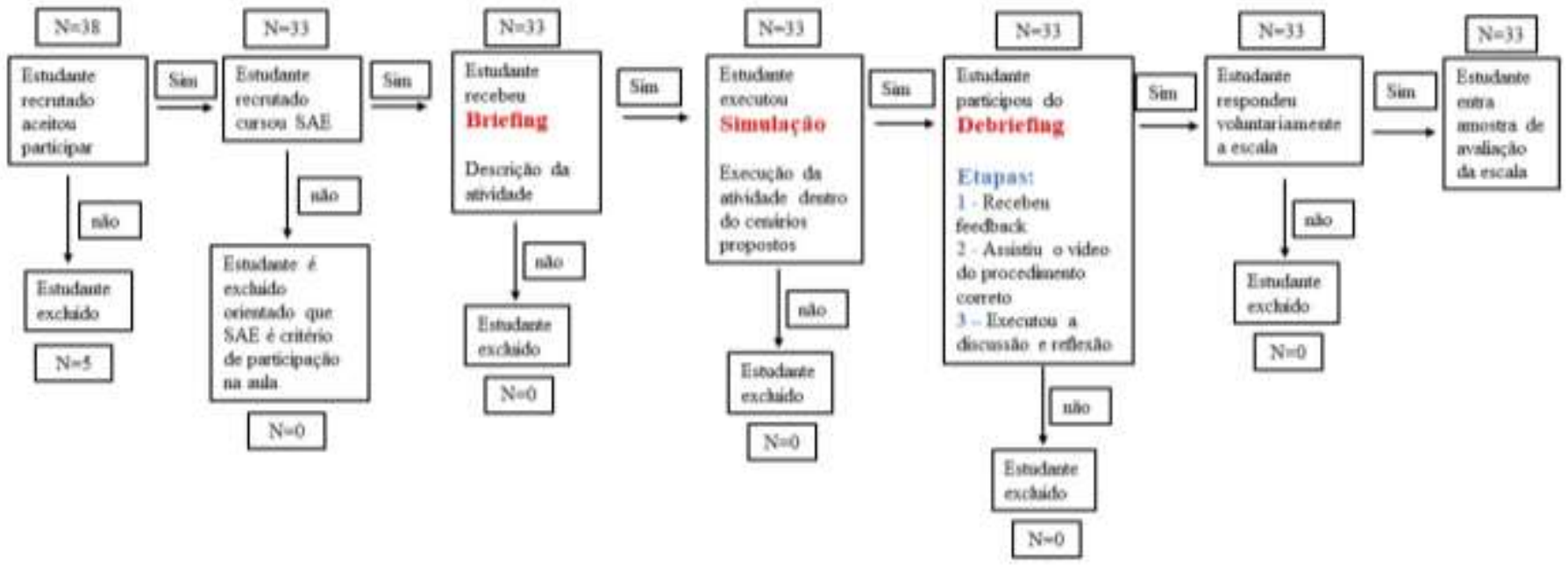

Fonte: Autores.

O estudo foi aprovado pelo Comitê de Ética em Pesquisa da Universidade Federal de Uberlândia sob o parecer 1864940. Aos participantes da pesquisa, foi solicitada a concordância por meio do Termo de Consentimento Livre e Esclarecido, sendo garantido o anonimato e o direito de desistência em qualquer fase da mesma.

\subsection{Caracterização da aula experimental}

A aula experimental se constituiu em uma aula experiencial baseada em simulação de situações realísticas, em três etapas sendo estas briefing, simulação e debriefing. Esta aula ocorreu no mesmo dia e antes da coleta de dados do perfil e da escala. O local da aula foi no laboratório de técnicas e habilidades multidisciplinares do Curso de Graduação Enfermagem da Faculdade de Medicina da Universidade Federal de Uberlândia - Minas Gerais, em 09 de novembro de 2017, após os 
participantes serem orientados quanto ao objetivo do estudo e assinarem o Termo de Consentimento Livre e Esclarecido. Os seguintes objetivos de aprendizagem foram estabelecidos para a aula: Ao final da simulação os estudantes deveriam chegar a pelo menos um diagnóstico de enfermagem prioritário entre os dois previamente estabelecidos. Alguns detalhes da construção da aula podem ser acessados em de Freitas Cortes, et al., (2019).

Para aplicação desta simulação, previamente os dois professores responsáveis pela disciplina SAE prepararam o laboratório de habilidades de enfermagem, com dois cenários idênticos, com a presença de dois atores, que foram treinados previamente e simularam um paciente com sintomas respiratórios. A turma foi dividida em quatro grupos, sendo que um aluno de cada grupo participou da simulação e os demais observaram a cena por meio de um vidro, permitindo apenas que os alunos observassem o que estava acontecendo dentro do cenário. Esta observação do cenário ocorreu em silêncio e sem que os alunos se manifestassem, de forma que o aluno que simulava não conseguisse ouvir quem estava na parte de trás do vidro e não sofresse interferência externa.

No primeiro momento denominado briefing, dois professores responsáveis pela disciplina SAE realizaram o briefing da simulação descrevendo toda a atividade de forma clara e objetiva (Araújo \& Quilici, 2012) para os dois grupos. Nessa etapa, os professores entregaram um formulário de descrição do cenário ao aluno que participou da simulação. Cabe destacar que o cenário foi previamente validado junto ao comitê de juízes e já está definido para ser utilizado junto à disciplina de SAE na instituição. Os demais alunos que observaram a simulação receberam um check list, com todos os passos do exame físico do sistema respiratório, construído segundo o referencial teórico de Barros, et al., (2015) e além de apontar as falhas no exame físico do sistema respiratório tinham também o objetivo de aprendizagem de encontrarem pelo menos um diagnóstico de enfermagem prioritário para o caso descrito.

No segundo momento aconteceu a simulação propriamente dita, dentro do cenário que foi previamente montado para que somente um estudante do grupo executasse a anamnese e o exame físico do sistema respiratório. Ao final da simulação o aluno deveria formular corretamente pelo menos um diagnóstico de enfermagem prioritário relacionado ao caso clínico apresentado. Foram eles o "Padrão respiratório ineficaz" relacionado à fadiga da musculatura respiratória evidenciado por ortopneia, padrão respiratório anormal ou a "Troca de gases prejudicada" relacionado à desequilíbrio na relação ventilaçãoperfusão evidenciado por respiração anormal (ex: frequência, ritmo, profundidade.) seguindo a taxonomia (Herdman \& Kamitsuru, 2017).

A simulação ocorreu após um encontro presencial e teórico de 4 horas-aula sobre exame do aparelho respiratório. O tempo da intervenção foi de 100 minutos, sendo 15 minutos de reconhecimento do cenário, 30 para o desenvolvimento, e 45 minutos de debriefing.

No terceiro momento denominado debriefing foi dado o feedback aos alunos e utilizado um vídeo que foi filmado previamente, demonstrando a execução correta do exame físico do sistema respiratório e a obtenção dos diagnósticos de enfermagem prioritários propostos para o caso clínico apresentado (de Freitas Cortes, et al., 2019). Após isto os alunos realizaram uma discussão e reflexão sobre a simulação. Este momento, não se referiu a uma avaliação formal do aluno, mas sim, tratou-se de um momento de discussão e reflexão, conduzindo os estudantes a consolidar conhecimentos e mudar atitudes inadequadas. Nesse momento, foram discutidas as ações, atitudes, necessidades apresentadas e o modo de atuação dos estudantes no cenário e na cena.

\subsection{Escala de satisfação e autoconfiança}

Para a avaliação de satisfação e autoconfiança sobre a aula experimental foi utilizado o instrumento validado denominado Student Satisfaction and Self-Confidence in Learning (Escala de Satisfação de Estudantes e Autoconfiança na Aprendizagem) (dos Santos Almeida, et al., 2015). O mesmo foi utilizado somente após solicitação e autorização do uso dessa 
escala para fins de pesquisa. Segundo dos Santos Almeida et al. (2015), a escala é desenvolvida para mensuração da satisfação e autoconfiança adquirida através da simulação de alta-fidelidade e é composta por 13 itens do tipo Likert de 5 pontos, dividida em: satisfação ( 6 itens, itens 1 a 5 e 9) e autoconfiança na aprendizagem ( 8 itens, itens 6 a 8 e 10 a 13), adicionalmente calculamos a média dos 13 itens. A validação para o português desta escala foi realizada através de um estudo com 350 mulheres e 45 homens na faixa de 29 anos onde sua confiabilidade constatada através do alfa de Cronbach foi de 0,94 para a subescala de satisfação e 0,87 para a subescala de autoconfiança (dos Santos Almeida, et al., 2015).

\subsection{Análises estatísticas}

Os dados coletados foram codificados e digitados duplamente por pessoas distintas em planilhas do aplicativo Excel, validados e analisados no programa estatístico SPSS. Os dados foram apresentados em forma de frequência absoluta e relativa ou como média e erro padrão do intervalo de confiança a 95\% (IC95\%) para distribuição Z; dependendo da variável. A normalidade dos dados contínuos foi testada pelo teste de Kolmogorov-Smirnov. Os dados dos dois fatores da escala foram ajustados ao modelo de regressão linear múltipla, pelo método dos mínimos quadrados ordinários, com as variáveis preditoras o sexo, a idade e o número de períodos cursados completos ou incompletos (testado separadamente em semestres, e dicotomizado em $3^{\circ}$ período e em outros). O modelo completo inicial foi reduzido utilizando-se como critério de exclusão a probabilidade das estimativas dos parâmetros maior que $10 \%$, baseada no teste $F$. Para todas as análises, foi adotado significância de 5\%, exceto quando descrito.

\section{Resultados}

Participaram do estudo uma amostra de 33 alunos, sendo que nenhum estudante inscrito na aula experimental se recusou a participar de todas as etapas estudo (Figura 1). Houve predominou o sexo feminino com 78,8\% $(\mathrm{n}=26)$ dos participantes em comparação ao masculino com 21,2\% ( $\mathrm{n}=7$ ). A idade média dos estudantes foi de 20,91 anos (IC95\%: 20,26; 21,56), e tinham cursado em média 3,33 períodos completos ou incompletos (IC95\%: 3,06; 3,60).

Em relação a escala de satisfação e autoconfiança; ambos os constructos mostraram médias de 4,36 e 4,15 respectivamente; evidenciando valores altos para os dois constructos. Todos os itens da escala obtiveram média acima de quatro, com exceção do item seis (Estou confiante de que domino o conteúdo da atividade de simulação que meu professor me apresentou) com média 3,39; mesmo assim um valor acima do moderado. A média geral dos 13 itens da escala foi de 4,24. (Tabela 1)

Quando avaliada a capacidade preditiva do perfil para os fatores da escala as variáveis idade e período (independente se em semestres ou dicotomizada), não foram significativas em nenhum dos modelos nos dois fatores da escala. $O$ fator satisfação teve como preditor o sexo feminino com $B i=-0,451$ (IC95\% $=(-0,748 ;-0,155) ; t=-3,109 ; p=0,004)$ que representa uma menor satisfação relacionada ao sexo feminino, sendo a constante do modelo $B i=4,714(\operatorname{IC95\% }=(4,451 ; 4,977) ; t=36,576 ; p=<$ 0,001). O fator autoconfiança não apresentou nenhum preditor significativo entre os testados. 
Tabela 1. Análise descritiva da escala Student Satisfaction and Self-Confidence in Learning, aplicadas a estudantes de enfermagem que participaram de uma simulação sobre exame físico respiratório na Sistematização da Assistência de Enfermagem com uso de debriefing com vídeo ( $\mathrm{n}=33$ estudantes).

\begin{tabular}{|c|c|}
\hline Item (unidade: ponto) & 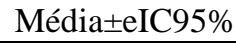 \\
\hline 1. Os métodos de ensino utilizados nesta simulação foram úteis e eficazes. & $4,33 \pm 0,16$ \\
\hline $\begin{array}{l}\text { 2. A simulação forneceu-me uma variedade de materiais didáticos e atividades para promover a } \\
\text { minha aprendizagem do currículo médico-cirúrgico. }\end{array}$ & $4,39 \pm 0,19$ \\
\hline 3. Eu gostei do modo como meu professor ensinou através da simulação. & $4,33 \pm 0,22$ \\
\hline 4. Os materiais didáticos utilizados nesta simulação foram motivadores e ajudaram-me a aprender. & $4,27 \pm 0,18$ \\
\hline $\begin{array}{l}\text { 5. A forma como o meu professor ensinou através da simulação foi adequada para a forma como eu } \\
\text { aprendo. }\end{array}$ & $4,30 \pm 0,23$ \\
\hline $\begin{array}{l}\text { 6. Estou confiante de que domino o conteúdo da atividade de simulação que meu professor me } \\
\text { apresentou. }\end{array}$ & $3,39 \pm 0,36$ \\
\hline $\begin{array}{l}\text { 7. Estou confiante que esta simulação incluiu o conteúdo necessário para o domínio do currículo } \\
\text { médico-cirúrgico. }\end{array}$ & $4,12 \pm 0,25$ \\
\hline $\begin{array}{l}\text { 8. Estou confiante de que estou desenvolvendo habilidades e obtendo os conhecimentos necessários a } \\
\text { partir desta simulação para executar os procedimentos necessários em um ambiente clínico. }\end{array}$ & $4,30 \pm 0,22$ \\
\hline 9. O meu professor utilizou recursos úteis para ensinar a simulação. & $4,52 \pm 0,17$ \\
\hline $\begin{array}{l}\text { 10. É minha responsabilidade como o aluno aprender o que eu preciso saber através da atividade de } \\
\text { simulação. }\end{array}$ & $4,42 \pm 0,27$ \\
\hline 11. Eu sei como obter ajuda quando eu não entender os conceitos abordados na simulação. & $4,33 \pm 0,27$ \\
\hline 12. Eu sei como usar atividades de simulação para aprender habilidades. & $4,15 \pm 0,28$ \\
\hline $\begin{array}{l}\text { 13. É responsabilidade do professor dizer-me o que eu preciso aprender na temática desenvolvida na } \\
\text { simulação durante a aula. }\end{array}$ & $4,30 \pm 0,38$ \\
\hline Média dos 13 itens & $4,24 \pm 0,12 ¥$ \\
\hline Constructo (unidade) & Média \pm eIC95\% \\
\hline Satisfação (ponto) & $4,36 \pm 0,13 ¥$ \\
\hline Autoconfiança (ponto) & $4,15 \pm 0,16 ¥$ \\
\hline
\end{tabular}

Legenda: eIC95\%: erro do intervalo de confiança a 95\%, obtido pela distribuição Z; ¥: valores médios seguidos pelo símbolo seguem distribuição normal pelo teste de Kolmogorov-Smirnov a 5\%. Fonte: Autores.

\section{Discussão}

A satisfação e a autoconfiança dos estudantes com relação ao processo de ensino-aprendizagem são construtos relevantes dentro de um ambiente de ensino (dos Santos Almeida, et al., 2015). Mensurá-los pode ser um forte indicativo para a utilização e avaliação de estratégias de ensino melhores e mais eficazes (dos Santos Almeida, et al., 2015). Nesse sentido, o presente estudo identificou que a aula experimental utilizando uma simulação realística com um vídeo no debriefing promove satisfação e autoconfiança na aprendizagem entre os estudantes de Enfermagem.

Costa, et al. (2020) comparou por meio da Escala de Satisfação de Estudantes e Autoconfiança na Aprendizagem, a metodologia tradicional com a simulação, e encontrou na simulação médias superiores, na maioria das variáveis e de ambas as subescalas. Além disso, observa-se que tais resultados coadunam com os deste estudo, sendo que em dois itens o presente estudo foi superior a 4,30; item cinco, "A forma como o meu professor ensinou por meio da simulação foi adequada para a forma como eu aprendo" e item onze, "Eu sei como obter ajuda quando eu não entender os conceitos abordados na simulação". Tais itens demonstram a satisfação dos estudantes quanto à condução da formação e aonde podem obter ajuda, ou seja, a forma como o professor desenvolve as etapas da simulação no sentido de ser um facilitador na aprendizagem de maneira não enviesada.

Em um estudo onde participaram estudantes do curso de graduação em enfermagem e foram utilizados questionários de práticas educacionais, design de simulação e satisfação dos alunos e autoconfiança. A escala de satisfação e autoconfiança apresentou resultados semelhantes ao do presente estudo, apontando que a maioria dos alunos concordou que estavam satisfeitos com a simulação e tinham autoconfiança para lidar com casos semelhantes no ambiente clínico (Al Khasawneh, et al., 2021). 
$\mathrm{Na}$ Noruega, uma pesquisa que teve como objetivo identificar os elementos da simulação baseada em cenários associados à satisfação e autoconfiança dos estudantes de enfermagem no gerenciamento da situação do paciente simulado constatou que s escores médios de autoconfiança e satisfação foram 4,16 e 4,57, respectivamente (Olaussen, et al. 2020). Eles observaram que estudantes o nível de fidelidade no cenário foi considerado elevado devido à imersão dos estudantes como estudantes autônomos na tomada de decisão e no desenvolvimento dos seus conhecimentos. Em outro estudo concluiu-se que a utilização de simulações em série, que sejam realizadas mais de uma vez em anos consecutivos é um método valioso de instrução clínica, ou seja, quando bem conduzidas, as simulações podem aumentar a satisfação e a autoconfiança dos estudantes (Zapko, et al., 2018). Al Khasawneh, et al. (2021) reforçam em seu estudo a relevância da simulação, recomendando fortemente a integração da simulação no currículo dos cursos de enfermagem. Desta maneira, destaca-se a importância de se inserir nos currículos de graduação em Enfermagem a simulação com metodologia de ensino, alcançando melhores resultados no processo de formação dos estudantes, para estarem aptos a exercerem uma prática reflexiva, crítica e comprometida, além de promover a autonomia, a liberdade, o diálogo e a resiliência perante os conflitos.

Neste estudo utilizou-se no debriefing um vídeo que demonstrava os passos do exame físico do sistema respiratório, o qual mostrou ser um recurso que reforçou as reflexões e o processo de ensino-aprendizagem. Em um estudo realizado com estudantes de final de curso de Enfermagem em uma universidade dos Estados Unidos, concluiu que o uso de desdobramento de cenários de pacientes em vídeo na sala de aula pode promover o envolvimento do aluno na aprendizagem (Powers, 2020). O uso do vídeo como um componente da simulação, tem mostrado ser uma ferramenta promissora, os resultados sugerem que o uso a simulação de vídeo em sala de aula pode aprofundar a compreensão dos alunos sobre o conteúdo ministrado em sala de aula e oferecer uma oportunidade a mais de aprendizagem para aperfeiçoar a aula expositiva (Herron, et al., 2019).

No item 4 "Os materiais didáticos utilizados nesta simulação foram motivadores e ajudaram-me a aprender" recebeu média 4,27, que mostra a relevância dos recursos didáticos que foram utilizados neste estudo, pois além do cenário construído para a simulação, a utilização deste vídeo que foi produzido especialmente para o momento do debriefing, foi muito relevante. Neste sentido, evidências científicas reforçam a importância do debriefing realizado após os cenários de simulação clínica, estudo quantitativo, realizado com estudantes de enfermagem, concluiu que o debriefing foi uma atividade reflexiva que contribuiu para que o estudante integrasse uma multiplicidade de saberes em valores afetivos, cognitivos e psicossociais, e desta forma, desenvolver as competências necessárias (Bortolato-Major, et al., 2019). Assim, reforça-se que o debriefing é uma parte fundamental no processo de simulação (Coutinho, et al., 2014).

Quanto ao item seis e sete, observam-se as pontuações mais baixas obtidas nesse estudo, o item seis: "Estou confiante de que domino o conteúdo da atividade de simulação que meu professor me apresentou e o item sete: "Estou confiante e que esta simulação incluiu o conteúdo necessário para o domínio do currículo de sistematização da assistência de enfermagem”. Esses itens se referem à procura pelo conhecimento, a aquisição de habilidades, a autonomia, a ajuda, e outros elementos que contribuem para o desenvolvimento de estudantes mais autoconfiantes.

No item oito, "Estou confiante de que estou desenvolvendo habilidades e obtendo os conhecimentos necessários a partir desta simulação para executar os procedimentos essenciais em um ambiente clínico", a média foi 4,3, o que aponta a importância da simulação no desenvolvimento do pensamento reflexivo e crítico, de forma, a trabalhar para minimizar as dicotomias que estão presentes nos programas de enfermagem, ou seja, reduzir a lacuna entre o aluno passivo do processo ensino-aprendizagem e o profissional ativo que o mercado de trabalho exige, que vive numa busca incansável por informações, que seja proativo, criativo, analítico, dinâmico, com raciocínio lógico e autonomia, capaz de ajudar na resolução dos problemas de saúde (JiménezGómez, et al.,2019). 
No que tange ao item dez: É minha responsabilidade como aluno aprender o que eu preciso saber através da atividade de simulação", apresentou média de 4,42; o que aponta para a responsabilidade do aluno perante sua aprendizagem, fornecendolhe autonomia perante o processo de ensino-aprendizagem.

Quanto aos itens doze e treze: "Eu sei como usar atividades de simulação para aprender habilidades. No item treze: "É responsabilidade do professor dizer-me o que eu preciso aprender na temática desenvolvida na simulação durante a aula. Nesse sentido, verifica-se que essas variáveis, são semelhantes ao estudo de Costa, et al., 2020, que sugere que essa média se justifica pela relevância da mediação do professor para orientá-los nos objetivos da aprendizagem. Essa autonomia pode ser compreendia como uma maneira de autoconfiança.

A menor satisfação das mulheres observada na subescala pode ter diversos efeitos causadores. As práticas educacionais (métodos de ensino e avaliação, bem como o desequilíbrio de gênero de muitos departamentos universitários) são destacados como impactantes especialmente no desempenho das mulheres e sugerem que as perspectivas de sujeito e gênero estão interagindo de maneiras que podem afetar a experiência e o desempenho dos alunos em todo o currículo universitário (Robson, et al. 2004). Outros aspectos que tem sido identificados sendo exemplos a qualidade do ensino, o curriculum ou até mesmo o uso de tecnologias afetam a percepção de satisfação em função do gênero (eg. Bean, Vesper, 1994; Dang et al. 2016). O papel dentro da simulação também pode afetar a satisfação de gêneros distintos como observado em estudantes de Medicina; com a discussão de que por exemplo o cenário de simulação pode ser mais atrativo aos homens (Tamás, et al. 2017). A Enfermagem é majoritariamente composta de mulheres, e não se esperaria tal resultado ou se esperaria uma satisfação menor entre os homens por representarem minoria. Ainda é necessário explorar porque e quais fatores estão associados a esse resultado; e não fomos capazes de acompanhar o papel do sujeito na simulação ou construir uma hipótese que justifique tal resultado; mas fica evidente a necessidade de compreender o papel de gênero no cenário de simulação.

\section{Conclusão}

Conclui-se que a aula experimental utilizando uma simulação realística com vídeo com a realização do procedimento correto no debriefing promove satisfação e autoconfiança na aprendizagem entre os estudantes de enfermagem, tendo em vista que todas as médias obtidas nos constructos de satisfação e autoconfiança da escala aplicada foram acima de quatro pontos. O que mostra que o uso da simulação realística possibilita uma prática reflexiva, crítica e que promove a autonomia dos estudantes de Enfermagem.

Em relação, as limitações deste estudo têm-se o tamanho da amostra, o uso de um perfil restrito, o não acompanhamento dos papeis e dos desfechos de resultado da simulação, a não replicação em diferentes instituições, a ausência de controle e a especificidade do tema abordado. Por outro lado, os dados reforçam o papel da simulação e que estudos futuros poderão contribuir para consolidar a relevância da simulação nos currículos de graduação em Enfermagem e definir e explicar quais seus fatores determinantes.

\section{Referências}

Al Gharibi, K. A., \& Arulappan, J. (2020). Repeated Simulation Experience on Self-Confidence, Critical Thinking, and Competence of Nurses and Nursing Students: An Integrative Review. SAGE open nursing, 6, 2377960820927377.

Al Khasawneh, E., Arulappan, J., Natarajan, J. R., Raman, S., \& Isac, C. (2021). Efficacy of Simulation Using NLN/Jeffries Nursing Education Simulation Framework on Satisfaction and Self-Confidence of Undergraduate Nursing Students in a Middle-Eastern Country. SAGE open nursing, 7, 23779608211011316.

Ali, A. A., Miller, E., Ballman, K., Bakas, T., Geis, G., \& Ying, J. (2020). The impact of debriefing modalities on nurse practitioner students' knowledge and leadership skills in managing fatal dysrhythmias: A pilot study. Nurse education in practice, 42, 102687.

Araújo, A. L. L. S., \& Quilici, A. P. (2012). O que é simulação e por que simular. ARAÚJO, ALLS,; QUILICI, AP Simulação Clínica: do conceito à aplicabilidade. ed. Atheneu. São Paulo. 
Baptista, R. C. N., Martins, J. C. A., Pereira, M. F. C. R., \& Mazzo, A. (2014). Students' satisfaction with simulated clinical experiences: validation of an assessment scale. Revista latino-americana de enfermagem, 22(5), 709-715.

Barros, A. L. B. L. Anamnese e exame físico: avaliação diagnóstica de enfermagem no adulto. (2015). Porto Alegre: Artmed, 472.

Bean, J. P., \& Vesper, N. (1994). Gender Differences in College Student Satisfaction. ASHE Annual Meeting Paper.

Bortolato-Major, C., Mantovani, M. D. F., Felix, J. V. C., Boostel, R., Silva, Â. T. M. D., \& Caravaca-Morera, J. A. (2019). Avaliação do debriefing na simulação clínica em enfermagem: um estudo transversal. Revista Brasileira de Enfermagem, 72(3), 788-794.

Brannan, J. D., White, A., \& Long, J. (2016). Learning styles: Impact on knowledge and confidence in nursing students in simulation and classroom. International journal of nursing education scholarship, 13(1), 63-73.

Costa, R. R., Medeiros, S. M., Coutinho, V. R., Mazzo, A., \& Araújo, M. S. (2020). Satisfação e autoconfiança na aprendizagem de estudantes de enfermagem: Ensaio clínico randomizado. Escola Anna Nery, 24(1), e20190094.

Coutinho, V. R. D., Martins, J. C. A., \& Pereira, M. D. F. C. R. (2014). Construção e validação da Escala de Avaliação do Debriefing associado à Simulação (EADaS). Revista de Enfermagem Referência, 4(2), 41-50.

Dang, Y. M., Zhang, Y. G., Ravindran, S., \& Osmonbekov, T. (2016). Examining student satisfaction and gender differences in technology-supported, blended learning. Journal of Information Systems Education, 27(2), 119.

de Freitas Cortes, C., da Silva Medeiros, N. A., da Silva, P. C. D. S., de Araújo, S. A., Oliveira, L. F., de Almeida Júnior, E. R., ... \& de Oliveira Dias, D. W. (2019). Relato de experiência sobre a elaboração e construção de vídeo-aula para o ensino do exame físico respiratório. Revista Eletrônica Acervo Saúde, 11(16), e1253-e1253.

dos Santos Almeida, R. G., Mazzo, A., Martins, J. C. A., Baptista, R. C. N., Girão, F. B., \& Mendes, I. A. C. (2015). Validation to Portuguese of the Scale of Student Satisfaction and Self-Confidence in Learning. Revista Latino-Americana de Enfermagem, 23(6), 1007.

Herdman TH, Kamitsuru S. NANDA International nursing diagnoses: Definitions classification, 2018-2020. New York: Theime; 2017. http://dx.doi.org/10.1055/b-006-161141

Herron, E. K., Powers, K., Mullen, L., \& Burkhart, B. (2019). Effect of case study versus video simulation on nursing students' satisfaction, self-confidence, and knowledge: A quasi-experimental study. Nurse education today, 79, 129-134.

Jiménez-Gómez, M. A., Cárdenas-Becerril, L., Velásquez-Oyola, M. B., Carrillo-Pineda, M., \& Barón-Díaz, L. Y. (2019). Reflective and critical thinking in nursing curriculum. Revista latino-americana de enfermagem, 27.

Johnsen, H. M., Fossum, M., Vivekananda-Schmidt, P., Fruhling, A., \& Slettebo, A. (2016). Teaching clinical reasoning and decision-making skills to nursing students: Design, development, and usability evaluation of a serious game. International journal of medical informatics, 94, 39-48.

Marmol, M. T., Braga, F. T. M. M., Garbin, L. M., Moreli, L., dos Santos, C. B., \& de Carvalho, E. C. (2012). Curativo de cateter central em simulador: efeito da presença do tutor ou da aprendizagem autoinstrucional. Revista Latino-Americana de Enfermagem, 20(6), 1134-1141.

Montejano-Lozoya, R., Gea-Caballero, V., Miguel-Montoya, I., Juárez-Vela, R., Sanjuán-Quiles, Á., \& Ferrer-Ferrandiz, E. (2019). Validation of a questionnaire designed to measure nursing student satisfaction with practical training. Revista latino-americana de enfermagem, 27.

Olaussen, C., Heggdal, K., \& Tvedt, C. R. (2020). Elements in scenario-based simulation associated with nursing students' self-confidence and satisfaction: A cross-sectional study. Nursing open, 7(1), 170-179.

Oliveira, S. N.; Prado, M. L.; Kempfer, S. S. Utilização da simulação no ensino da enfermagem: revisão integrativa, (2014). Revista Mineira de Enfermagem, 18(2), 487-504.

Padilha, J. M., Machado, P. P., Ribeiro, A., Ramos, J., \& Costa, P. (2019). Clinical virtual simulation in nursing education: randomized controlled trial. Journal of medical Internet research, 21(3), e11529.

Polit, D. F., \& Beck, C. T. (2006). The content validity index: are you sure you know what's being reported? Critique and recommendations. Research in nursing \& health, 29(5), 489-497.

Powers, K. (2020). Bringing simulation to the classroom using an unfolding video patient scenario: A quasi-experimental study to examine student satisfaction, self-confidence, and perceptions of simulation design. Nurse education today, 86, 104324.

Robson, J. \& Francis, B. \& Read, B. (2004). Gender, student confidence and communicative styles at university: The views of lecturers in history and psychology. Studies in Higher Education - Studies in Higher Education. 29. 7-23.

Tamás, É., Edelbring, S., Hjelm, C., Hult, H., \& Gimm, O. (2017). Gender and Assigned Role Influences Medical Students' Learning Experience in Interprofessional Team Training Simulations. MedEdPublish, 6.

Zapko, K. A., Ferranto, M. L. G., Blasiman, R., \& Shelestak, D. (2018). Evaluating best educational practices, student satisfaction, and self-confidence in simulation: A descriptive study. Nurse education today, 60, 28-34. 